\title{
The effect of femoral nerve block on fracture healing via expressions of growth factors and $\beta$-catenin
}

\author{
Serap Uslu ${ }^{1}$, Arzu Gercek Irban², Arel Gereli ${ }^{3}$, Elif Ilgaz Aydinlar ${ }^{4}$, \\ Pinar Elpen ${ }^{5}$, Umit Ince ${ }^{6}$
}

${ }^{1}$ Department of Histology and Embryology, Medical School, Istanbul Medeniyet University, Istanbul, Turkey

${ }^{2}$ Department of Anesthesiology and Reanimation, Medical School, Medipol University, Istanbul, Turkey

${ }^{3}$ Department of Orthopedic and Traumatology, Medical School, Acibadem University, Istanbul, Turkey

${ }^{4}$ Department of Neurology, Medical School, Acibadem University, Istanbul, Turkey

${ }^{5}$ Vocational School of Radiology Program, Acibadem University, Istanbul, Turkey

${ }^{6}$ Department of Pathology, Medical School, Acibadem University, Istanbul, Turkey

\begin{abstract}
Introduction. Many patients of all ages are admitted to hospital due to bone fractures. The etiology of fracture has a very wide spectrum, ranging from motor accidents to pathological conditions such as tumors, osteoporosis, and others. Bone fracture healing is a well-programmed and well-organized process, but is also long and intractable. The outcome of this process is therefore affected by many factors, such as the patient's age, ethnicity, nutritional status, and extent of the fracture. At present, regional analgesic techniques are frequently applied in order to avoid the complications of systemic opioid administration, central block applications. Femoral block is one of the regional analgesic techniques frequently applied by anesthesiologists when the lower extremities are involved. In this study, we evaluated the effect of femoral nerve block on the healing of an experimental non-stabilized femur fracture via expression of TGF- $\beta$, VEGF, and $\beta$-catenin and bone histomorphometry in rats. Material and methods. In the control group, only the femoral fracture was performed and the bone was not fixated, similarly as in other groups. In the One-Day Block group, a one-time femoral nerve block was applied after the femoral fracture. In the Three-Day Block group, a daily femoral nerve block was performed for three days after the femoral fracture. On Days 4, 7, and 13, femurs were excised. The bone sections were stained with hematoxylin-eosin to evaluate bone tissue and Safranin $O$ to assess callus tissue, cartilaginous tissue, and new bone areas. TGF- $\beta$, VEGF, and $\beta$-catenin were assessed by immunohistochemistry.

Results. Histomorphometric analysis revealed that femoral block application had a positive impact on bone healing. TGF- $\beta$ expression in the One-Day and Three-Day Block Groups was significantly higher than in the control group at all times, as was also the case with VEGF expression. On day $13, \beta$-catenin expression was significantly higher in the Three-Day Block group than the others.

Conclusions. The results of the study suggests that the applications of a femoral nerve block for perioperative analgesia, for either one day or three days, resulted in better and more rapid bone healing. (Folia Histochemica et Cytobiologica 2016, Vol. 54, No. 3, 151-158)
\end{abstract}

Key words: fracture healing; TGF- $\beta$; VEGF; $\beta$-catenin; histomorphometry; femoral nerve block; rat

Correspondence address: S. Uslu, Assist. Prof., Ph.D.

Department of Histology and Embryology

Istanbul Medeniyet University Medical School

Istanbul, Turkey

tel.: 90532325 8849, e-mail: musiuslu@gmail.com 


\section{Introduction}

Patients of all ages are admitted to hospital due to bone fractures in great numbers. The etiology of fracture covers a very wide spectrum, from motor accidents to pathological conditions such as tumors, osteoporosis, and so on. Bone fracture healing is a well-programmed and well-organized process, but it is also long and intractable. The outcome of the process is thus affected by many factors, such as patient's age, ethnicity, nutritional status, physical condition, and the etiology and extent of the fracture [1-5]. The healing process following bone fracture takes one of two different paths, depending on whether or not a stabilization procedure was performed: non-stabilized fractures heal via endochondral ossification, while stabilized fractures heal via intramembranous ossification [6]. The process of endochondral ossification begins with the proliferation and differentiation of mesenchymal cells (MSCs) into cartilage in the following six stages: hematoma formation and inflammation, angiogenesis, formation of cartilage, calcification of cartilage, cartilaginous transformation into bone, and remodeling $[1,7]$.

Many key regulating factors and signaling pathways affect bone repair; these include fibroblast growth factors (FGFs), platelet-derived growth factors (PDGFs), transforming growth factor beta (TGF- $\beta$ ), vascular endothelial growth factors (VEGFs), and bone morphogenetic proteins (BMPs) $[4,5]$. TGF- $\beta$ has direct effects on the regulation of osteoblast differentiation; its reduction in osteoblasts has been shown to expand bone mineral concentration and bone mass [8-10]. VEGF, a known promoter of angiogenesis, stimulates neovascularization and promotes fracture healing, and also regulates activity of osteoclasts and osteoblasts [11, 12]. The formation of new blood vessels (angiogenesis) plays an important role during bone fracture healing since vascular changes precede bone formation [13]. $\beta$-catenin is essential in determining whether MSC progenitors become osteoblasts and suppress osteoclasts $[4,14,15]$.

Nowadays, regional analgesic techniques are frequently applied in order to avoid the complications of systemic opioid administration, central block applications, to supply better perioperative analgesia and reduce costs and duration of hospitalization [16]. Femoral block is one of the regional analgesic techniques frequently applied by anesthesiologists when the lower extremities are involved [16-18].

The aim of this study was to evaluate the effect of femoral nerve block on the healing of experimental non-stabilized femur fractures via the assessment of the expression of TGF- $\beta$, VEGF, and $\beta$-catenin and bone histomorphometry in rat.

\section{Material and methods}

Experimental and animal design. All experiments were approved by the local Animal Ethics Committee at Marmara University School of Medicine, Istanbul, Turkey. To investigate the effects of femoral nerve block on fracture healings in rat, a total of 72 male rats, each weighing between 120 and $160 \mathrm{~g}$, were divided randomly into three groups using sealed envelopes. In all groups, the animals were anesthetized by intramuscular injection of xylazine $(50 \mathrm{mg} / \mathrm{kg}$, Bayer United German Pharmaceutical Factories, Istanbul, Turkey) and ketamine hydrochloride (50 mg/kg, Parke-Davis, Istanbul, Turkey).

Femoral fracture (ffx) technique. The femoral fractures were performed in line with the description of Le et al. [19]. After shaving the right leg, surgical fields were draped under sterile conditions. Avertical lateral incision at the femur was followed by muscular blunt dissection. The femur bone was fractured transversally with a manual bone cutter at the level of diaphysis. The bone was not fixated. The surgical wound was closed with a nonabsorbable suture. On Days 4,7 , and 13 , eight rats from each group were euthanized by decapitation. The right femurs were then excised.

Radiographic imaging. The rats were processed immediately after radiographic examination. Radiographic analysis was performed using a Siemens syngo fastView (2011, Siemens, Erlangen, Germany) while the rats were still under the effect of anesthetic drugs. The rats were placed first in the prone position and then in the lateral position. Their right legs were abducted. The X-ray images were taken at $45 \mathrm{kV}, 2 \mathrm{mAS}$. The images were examined and the fractured bones were standardized (Figure 1).

Femoral nerve block (FNB) technique. Femoral nerve block (FNB) was carried out using the landmarking technique. For injection the rat was held in lateral recumbency with the limb to be injected forming a right angle with the longitudinal axis of the trunk. The greater trochanter and ischial tuberosity were localized by palpation. On an imaginary line from the greater trochanter to the ischial tuberosity, about one third of the distance caudal to the greater trochanter, the injection needle was advanced from dorsolateral direction at a $45^{\circ}$ angel until the tip encountered the ischium [20]. $0.1 \mathrm{~mL}$ of $0.25 \%$ levobupivacaine (Chirocaine, Nycomed Pharma AS, Elverum, Norway) was injected. The success of FNB technique was tested through the withdrawal response of the paw to a forceps pinch on the lateral foot/toe. The pinch was limited to a maximum of 1 second. The sensory responses were evaluated on the basis of no response to pinch at full sensory block. Following experimental groups were set up:

- Control group $(n=24)$ : only ffx was performed;

- One-Day Block group ( $\mathrm{n}=24)$ : after ffx was applied, FNB was performed for one day; 


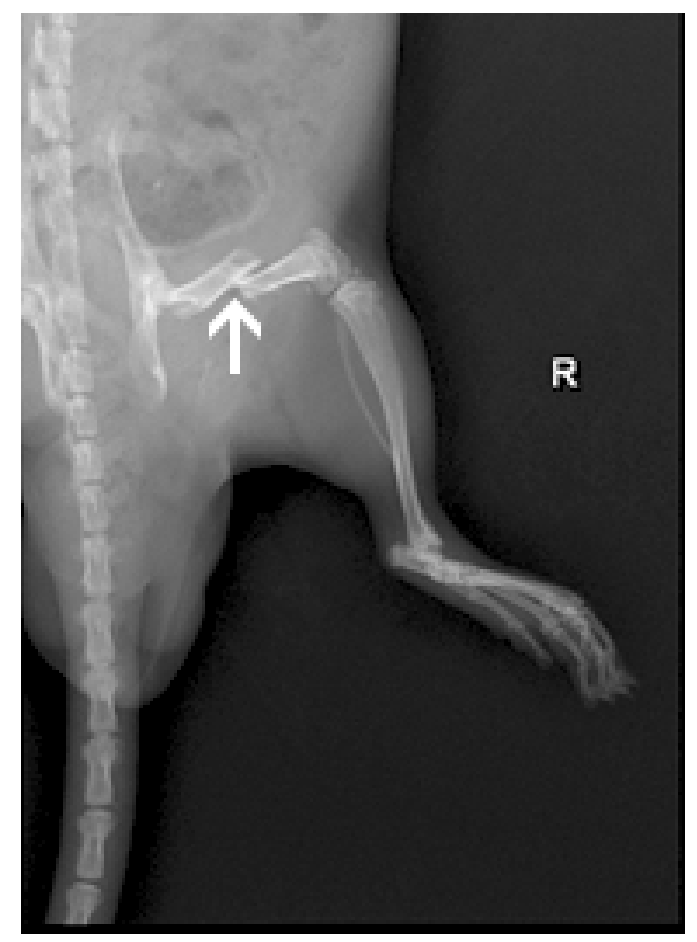

Figure 1. Radiographic image of nonstabilized femoral fracture of an anesthetized rat. The fracture was performed as described in Material and methods. Arrow shows site of fracture.

- Three-Days Block group $(\mathrm{n}=24)$ : after ffx was performed, FNB was applied as described above once a day for three consecutive days [17].

Additionally, $50 \mathrm{mg} / \mathrm{kg}$ ketorolac (Ketorolac trometamin, Deva, Istanbul, Turkey) was given to all rats intraperitoneally for postoperative analgesia for 5 days.

Histological analyses. Bone samples were fixed in $10 \%$ neutral buffered formalin for 24 hours and then decalcified in decalcifier solution (Shandon TBD-2 Decalcifier, Runcorn, UK) at room temperature for 5 days. Each specimen was dehydrated using a graded ethanol series and later cleared in xylene, before being embedded in paraffin. The paraffin blocks were cut into $5 \mu \mathrm{m}$ and the sections were mounted on glass slides. The sections were stained with hematoxylin acc. to Gill III and eosin (H\&E staining) (Merck, Darmstadt, Germany) to evaluate bone morphology and Safranin O (Merck) to evaluate the presence of callus tissue and cartilaginous and new bone areas.

Histomorphometric analyses. Five serial sections from each leg and a minimum of five adjacent fields in each section were quantified at a magnification of $\times 40$ objective lens. The thicknesses of the periosteum, number, thickness, and areas of trabeculae and cartilaginous new bone were evaluated histomorphometrically with a use of semiautomatic image analysis system (University of Texas Health Science Center at Santorino, TX, USA image tool for Windows version 1.28 program) as described by other authors [6, 21-24]. All of the measurements were made in a blinded manner by a histologist.

Immunohistochemistry. Bone tissue sections $5-\mu \mathrm{m}$-thick were obtained from the paraffin blocks. The tissue blocks were chosen carefully after histological assessment of the sections stained with H\&E. For immunohistochemical (IHC) staining, sections were incubated overnight at $60^{\circ} \mathrm{C}$ and then immersed in xylene and rehydrated in a decreasing series of ethanol solutions. The sections were washed with both distilled water and phosphate-buffered saline (PBS) solution (P4417; Sigma-Aldrich, St Louis, MO, USA) for 10 $\mathrm{min}$ and then treated with citrate buffer $(\mathrm{pH}: 7.6)$ for $5 \mathrm{~min}$ in a pressure cooker. Following washing with PBS, the sections were delineated with a Dako Pap pen (Dako, Glostrup, Denmark) and incubated in a solution of $3 \%$ hydrogen peroxide (Peroxidase Block) for $5 \mathrm{~min}$ to inhibit endogenous peroxidase activity. After washing in PBS, the sections were incubated with non-immune serum for $1 \mathrm{~h}$ and then incubated with primary antibodies: TGF- $\beta$ (1:100, Genetex, Irvine, CA, USA), VEGF (1:100, cat no GTX22992, Genetex), $\beta$-catenin (ready to use, cat no GTX15180, Genetex) overnight at $4^{\circ} \mathrm{C}$ in a humidity chamber. The sections were washed 3 times for 5 min each in PBS, followed by incubation with a post-primary rabbit anti-mouse $\mathrm{IgG}$ for $30 \mathrm{~min}$ and then with Novolink Polymer Anti-rabbit poly-HRP-IgG (Novocastra, RE715K, Newcastle, UK) for $30 \mathrm{~min}$. After washing 3 times with PBS, the sections were incubated with DAB substrate buffer for 5 min using the NovoLink polymer detection system (RE715K, Novocastra). After washing with distilled water the sections were counterstained with Mayer's hematoxylin and washed with distilled water. In the negative controls, the primary antibodies were omitted. All the sections were mounted with mounting medium (Shandon EZ-Mount, ThermoFisher Scientific, Waltham, MA, USA) and the immunoreactive cells were in Olympus BX51, bright-field microscope (Olympus, Tokyo, Japan). The presence of a brown precipitate indicated a positive reaction for primary antibodies. To determine the immunoreactivity of the sections, a series of semi-quantitative analyses were performed in the tissue materials. HSCORE was calculated using the following equation: $\mathrm{HSCORE}=\sum \mathrm{Pi}(\mathrm{I}+1)$, where ' $\mathrm{i}$ ' is the intensity of labeling with a value of 1,2 or 3 (weak, moderate, or strong, respectively) and $\mathrm{Pi}$ is the percentage of labelled cells for each intensity, varying from $0 \%$ to $100 \%$.

Statistical analyses. For statistical analysis non-parametric ANOVA test, Kruskall Wallis test and Dunn's test as a posthoc test were used $(\mathrm{p}<0.05)$. For this analysis GraphPad Instat program (Graphpad Software Inc., La Jolla, CA, USA) was applied. 


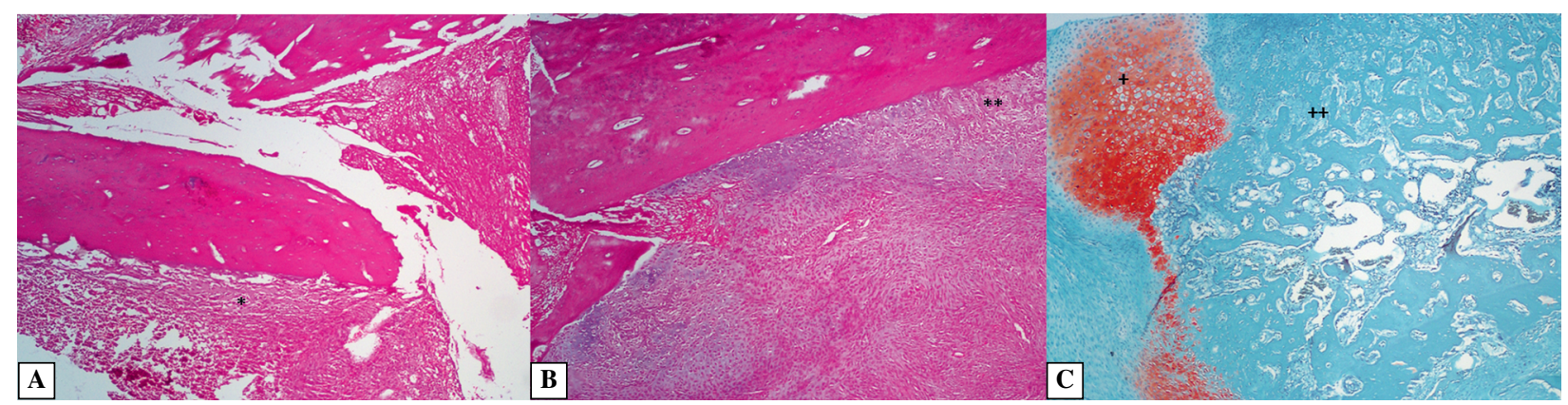

Figure 2. The histological appearance of fracture area after femoral block application. A. One-Day Block group: *periosteum, H\&E staining, $\times 40$; B. One-Day Block group: **compact and trabecular bone, H\&E staining, $\times 40 ;$ C. Three-Day Block group: +cancellous bone, ++ new bone formation, safranin $\mathrm{O}$ staining, $\times 40$.

Table 1. Histomorphometric analyses of fractured and healing femoral bone in rats subjected to femoral nerve blockade on days 4,7 and 13 after fracture

\begin{tabular}{|l|c|c|c|c|c|c|c|c|c|}
\hline & \multicolumn{3}{|c|}{ Day 4 } & \multicolumn{3}{c|}{ Day 7 } & \multicolumn{2}{c|}{ Day 13 } \\
\hline & $\begin{array}{c}\text { Control } \\
\text { group } \\
(\mathbf{n = 8})\end{array}$ & $\begin{array}{c}\text { 1-Day } \\
\text { group } \\
(\mathbf{n = 8})\end{array}$ & $\begin{array}{c}\text { 3-Day } \\
\text { group } \\
(\mathbf{n = 8})\end{array}$ & $\begin{array}{c}\text { Control } \\
\text { group } \\
(\mathbf{n}=\mathbf{8})\end{array}$ & $\begin{array}{c}\text { 1-Day } \\
\text { group } \\
(\mathbf{n = 8})\end{array}$ & $\begin{array}{c}\text { 3-Day } \\
\text { group } \\
(\mathbf{n}=\mathbf{8})\end{array}$ & $\begin{array}{c}\text { Control } \\
\text { group } \\
(\mathbf{n}=\mathbf{8})\end{array}$ & $\begin{array}{c}\text { 1-Day } \\
\text { group } \\
(\mathbf{n}=\mathbf{8})\end{array}$ & $\begin{array}{c}\text { 3-Day } \\
\text { group } \\
(\mathbf{n}=\mathbf{8})\end{array}$ \\
\hline $\begin{array}{l}\text { Periosteum } \\
\text { thickness }\end{array}$ & $34 \pm 8$ & $54 \pm 13^{*}$ & $60 \pm 13^{* * *}$ & & & & & & \\
\hline $\begin{array}{l}\text { Trabecular } \\
\text { number }\end{array}$ & & & & $16 \pm 5$ & $50 \pm 14^{* *}$ & $45 \pm 31^{*}$ & $19 \pm 5$ & $34 \pm 4^{* * *}$ & $24 \pm 4^{4 a a}$ \\
\hline $\begin{array}{l}\text { Trabecular } \\
\text { thickness }\end{array}$ & & & & $16 \pm 4$ & $17 \pm 4$ & $18 \pm 4$ & $13 \pm 3$ & $16 \pm 3$ & $16 \pm 3$ \\
\hline $\begin{array}{l}\text { Trabecular } \\
\text { area }\end{array}$ & & & $1120 \pm 220$ & $1356 \pm 260$ & $1766 \pm 350^{* * *, a}$ & $782 \pm 150$ & $1259 \pm 240^{* * *}$ & $1335 \pm 265^{* * *}$ \\
\hline $\begin{array}{l}\text { Cartilaginous } \\
\text { area }\end{array}$ & & & $21134 \pm 2825$ & $16734 \pm 1006^{* * * *}$ & $16123 \pm 1209^{* * *}$ & $91885 \pm 1393$ & $96164 \pm$ & $77047 \pm$ \\
\hline $\begin{array}{l}\text { New bone } \\
\text { area }\end{array}$ & & & $68899 \pm 3809$ & $104910 \pm 4209^{*}$ & $84145 \pm 4530^{* * *}$ & $136192 \pm 2013$ & $131705 \pm 4803$ & $189980 \pm 4347$ \\
\hline
\end{tabular}

Data present mean $\pm \mathrm{SD}, \mathrm{n}=24 .{ }^{*}, * *, * * * \mathrm{p}<0.05, \mathrm{p}<0.01$, and $\mathrm{p}<0.001$, respectively, when compared with the same day Control group; a, aaa $p<0.05$ and $\mathrm{p}<0.001$ for the same day values between the One-Day and Three-Day Block groups. All thickness values are expressed in pixels $(1$ pixel $=0.36 \mu \mathrm{m})$ and all area values in pixel$^{2}$.

\section{Results}

\section{Effects of femoral fracture and femoral nerve block on the histomorphometric parameters of rat femur} Periosteal thickness (Ps.Th): On Day 4, a significant increase was observed in the thickness of the periosteum of the One-Day block group and the Three-Day block group over that of the Control group ( $p<0.05$ and $\mathrm{p}<0.01$ respectively) (Figure $2 \mathrm{~A}$, Table 1 ).

Trabecular number (Tb.N): On Day 7, a significant increase was seen in the trabecular numbers per area in the One-Day block group $(\mathrm{p}<0.01)$ and the Three-Day block group $(\mathrm{p}<0.05)$, as compared with the Control group. When we compared the Control group and the One-Day block group on Day 13, we found a significant increase in the One-Day block group ( $\mathrm{p}<0.001)$. It was also observed that there was a decrease in trabecular numbers between the OneDay block and Three-day block groups $(\mathrm{p}<0.001)$ (Figure 2B, Table 1).

Trabecular thickness (Tb.Wi): We could not find any statistical differences in the trabecular thickness between the groups $(\mathrm{p}>0.05)$ (Figure 2B, Table 1).

Trabecular area (Tb.Ar): When we compared the One-Day Block with the Control group, we found an increase in the trabecular area, but this increase was not statistically significant by Day $7(p>0.05)$. There were statistically significant increases in the ThreeDay block group compared with the Control group $(p<0.001)$, and it was also observed that there was an increase in the trabecular area compared with the One-Day block group on Day $7(p<0.05)$. There was 

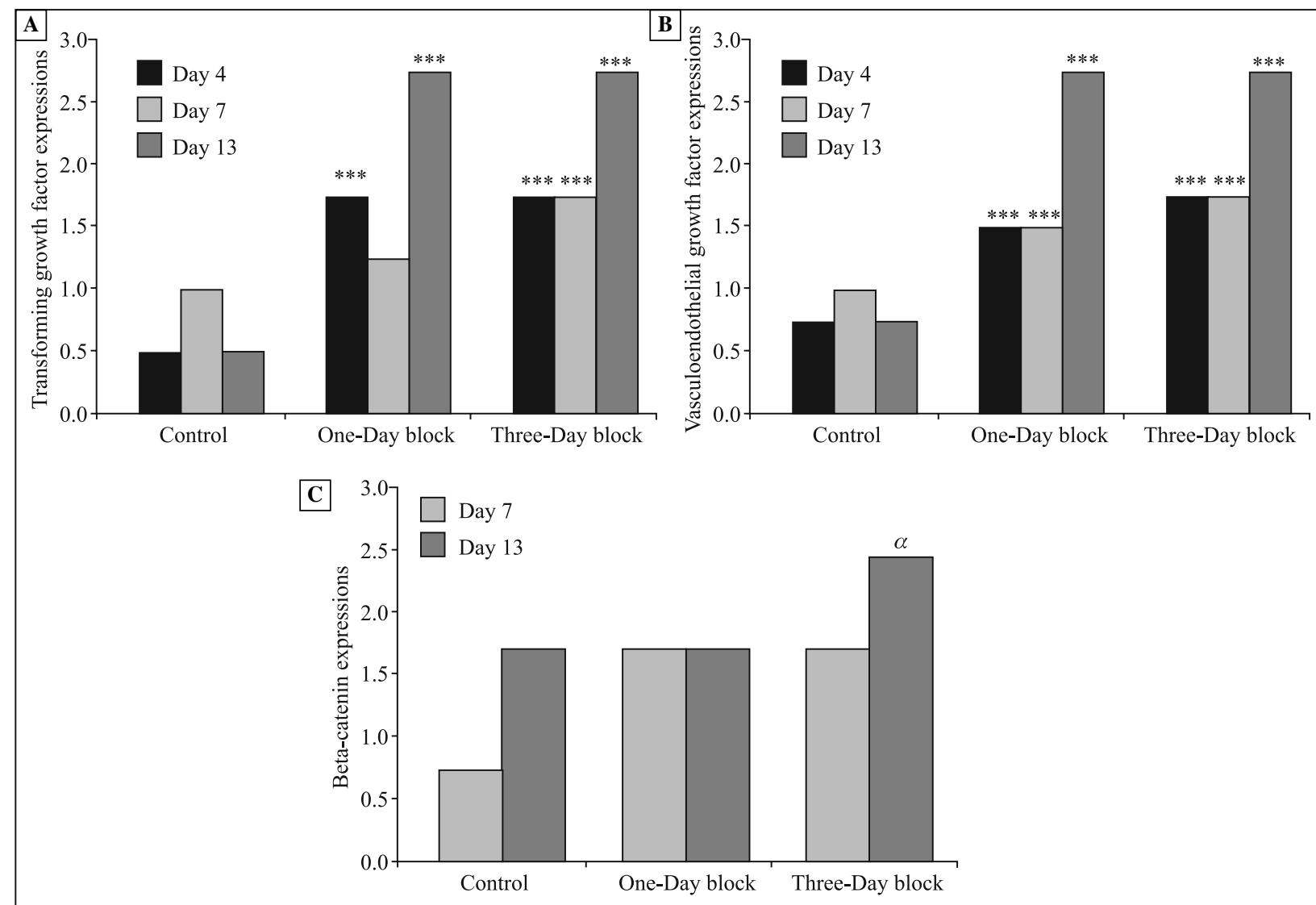

Figure 3. Comparison of the immunohistochemical expression of growth factors and $\beta$-catenin. Expression of TGF (A), VEGF (B) and $\beta$-catenin (C) in Control, One-Day Block and Three-Day Block Groups. *,**,***compared with Control group on the same day, $\mathrm{p}<0.05, \mathrm{p}<0.01$ and $\mathrm{p}<0.001$, respectively; ${ }^{\alpha}$ compared with the other groups on the same day, $\mathrm{p}<0.05$.

a significant increase in the One-Day and Three-Day block groups, as compared with the Control group on Day 13 (p < 0.001) (Figure 2B, Table 1).

Cartilaginous Area (Cg.Ar): There was a decrease in both the treatment groups on Day 7 compared with the Control group $(\mathrm{p}<0.001)$. When we compared the One-Day block group with the Control group, an increase was found in the One-Day Block group $(\mathrm{p}<0.001)$ and a decrease was found in the Three-Day Block group on Day 13 ( $\mathrm{p}<0.001)$. The cartilaginous areas had decreased in the Three-Day block group, as compared with the One-Day block group on Day 13 ( $\mathrm{p}<0.001)$ (Figure 2C, Table 1).

New bone area (B.Ar): The statistical analysis showed that the new bone area increased in both block groups on Day 7 ( $\mathrm{p}<0.001)$; in addition, the One-Day block group's new bone area decreased compared with the Control group, but this decrease was not statistically significant on Day 13 ( $p>0.005)$. The Three-Day block group's new bone area increased compared with the Control group and also increased compared with the One-Day block group on Day 13 $(\mathrm{p}<0.001)$ (Figure 2C, Table 1).

\section{Effects of ffx and femoral nerve block on the immunoexpression of TGF- $\beta, V E G F$, and $\beta$-catenin in rat femur}

The H-SCORE values of TGF- $\beta$, VEGF, and $\beta$-catenin immunoreactivity in fracture healing were analyzed (Figure 3A-C).

When compared with the control group, TGF- $\beta$ expression was found to be statistically higher both in the One-Day block group and in the Three-Day block group at all times ( $<0.001$ for all) (Figure 3A, Figure 4A1-A3).

When compared with the control group, there were significant increases in VEGF expressions in both groups at all times ( $p<0.001$ for all). There was no significant difference between the VEGF expression in the block groups on Day 4 or Day 13 (Figure 3B, Figure 4B1-B3).

There were increases on Day 7 in the $\beta$-catenin expression of the One-Day and of the Three-Day block groups over the Control group. However, there was a significant increase in the Three-Day block group on Day $13(\mathrm{p}<0.05) . \beta$-catenin expression was the same for the two block groups on Day 7, but in the 


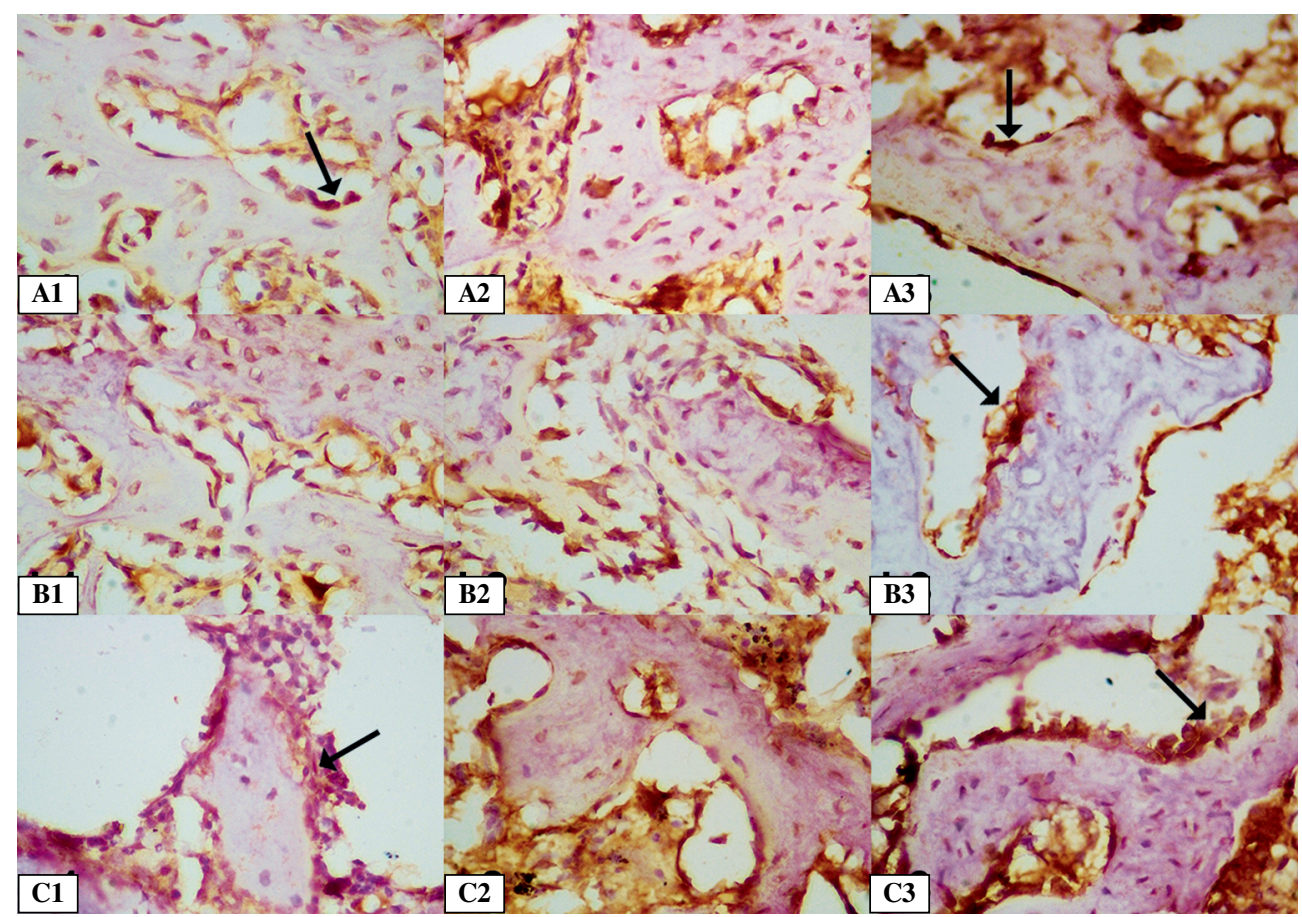

Figure 4. Immunohistochemical expression of TGF- $\beta$, VEGF and $\beta$-catenin around the trabeculae in the fracture area. A1-3. TGF- $\beta$ expression on Day 13 in Control, One-Day and Three-Day block groups, respectively; B1-3. VEGF expression on Day 13 in Control, One-Day and Three-Day block groups, respectively; C1-3. $\beta$-catenin expression on Day 13 in Control, One-Day and Three-Day block groups, respectively. Arrow shows osteoblast, magnification $\times 400$.

Three-Day block group, the expression had increased by Day 13 (Figure 3C, Figure 4C1-C3).

\section{Discussion}

By Day 3 after a fracture, when the fracture formation healing process starts, MSCs are recruited and begin to proliferate. They subsequently differentiate into chondroblasts. The proliferation of these new chondrocytes occurs from Day 7 to Day 21, resulting in formation of the soft callus. The periosteum is the most important factor at this stage. When the periosteum is removed, the fracture callus development is diminished, because better periosteal growth may lead to better endochondral ossification $[1,25]$. In our study, we showed that the periosteum thicknesses of the treatment groups were higher than those of the Control group on Day 4. Le et al. have shown that fracture healing in both nonimmobilized and immobilized rat fracture calluses, though different radiographically and histologically, is characterized by similar biochemical changes in the ground substance [19]. Although the callus that forms in the immobilized model is appreciably smaller than that formed when the fracture is not immobilized, the chemical composition of the organic matrix of the existing callus undergoes similar modifications, regardless of fixation or histological response.
After Day 7, the cartilaginous callus surrounding the fracture site has become large $[1,3,19]$. Vortkamp et al. claim that, by Day 14, the callus has been replaced by bone in the fracture area, so that only small cartilage regions remain [26]. Diniz et al. suggest that the persistence of a large cartilaginous callus, together with a trabecular bone with sparse spacing, imply delayed healing and less mature calluses in rodents [27]. In our study, the reduction of the cartilaginous area, and the increase in the new bone and trabecular area and number were identified; these positive effects depend on femoral block treatment after the fracture.

Previous studies show that TGF- $\beta$ a potent mitogen for bone-forming cells that controls the proliferation of undifferentiated mesenchymal and osteoprogenitor cells, osteoblasts, and chondrocytes - is expressed from an early stage in fracture healing. TGF- $\beta$ is secreted in the fracture site on Day 0 and secretion continues to Day 21 of the healing process. By Day 3, MSC proliferation and angiogenesis have begun as a result of TGF- $\beta$ expression. By Day 7, after fracture endochondral ossification has started, TGF- $\beta$ expression continues. By Day 14, TGF- $\beta$ expression is declining $[1,3,28]$. Our results show that TGF- $\beta$ expression in the femoral nerve block groups was found to be higher than in the Control group on Days 4, 7, and 13. 
The literature reveals that VEGF has been shown to stimulate bone healing in the fracture callus during repair in animal models [5, 28]. Kanczler et al. indicate that VEGF factor isoforms are essential in coordinating cartilage formation and ossification during endochondral bone development [29]. The stimulation of the circulation of endosteum a membrane lining the inner surface of the bone allows the MSCs associated with growing capillaries to invade the wound region from the endosteum and bone marrow. VEGF expression is detected in chondroblasts, chondrocytes, osteoprogenitor cells, and osteoblasts in the fracture callus, where it is highly expressed in angioblasts, osteoprogenitor, and osteoblast cells during the first seven days of healing, but decreases after eleven days, activating not only angiogenesis but also osteoclast recovery, differentiation, and activity. This leads to the remodeling of the fracture callus during endochondral ossification. Other studies indicate that VEGF expression starts on Day 14 after the fracture, and goes on throughout the remodeling process. Investigations of the tissue level report increased interaction between blood vessel formation and bone regeneration $[1,3,6]$. Kanczler et al. claim that the inhibition of VEGF in fractured mouse femurs results in a decrease in blood vessel invasion with a reduction in osteoclastic bone remodeling, impaired callus mineralization, and reduced trabecular bone healing [29]. Our study shows that the VEGF expression of the treatment groups increases after the application of femoral nerve block.

$\beta$-catenin has a major effect on the differentiation of MSCs to osteoblasts and chondroblasts in bone fracture healing. Studies have shown that the activation of $\beta$-catenin increases in the early period of endochondral ossification but decreases in its late period. When an osteoblast changes into an osteocyte, $\beta$-catenin expression decreases in the late period of differentiation. The expression of $\beta$-catenin increases on Day 3 and rises to a peak on Day 10, but cannot be detected in rats on Day $21[15,30]$. Our results suggest that the application of femoral nerve block for three days resulted in greater expression of $\beta$-catenin on Day 13 in the group that received it than in the others.

This study has revealed that, in the case of femoral fracture, the application of femoral nerve block accelerates the healing process of femoral fracture in rats. In addition to the humanitarian and economic aspects of effective pain management, the use of nerve block provides better postoperative pain control than systemic patient-controlled analgesia (PCA) [31,32], reduces the need for opioids and their related complications, allows earlier mobilization and functional recovery, and shortens stays at hospitals and reha- bilitation centers $[33,34]$. Most studies agree that opioid consumption decreases by $40-70 \%$ when nerve blocks are used, when compared with PCA alone. Another significant advantage of the use of nerve blocks in the ambulatory patient is that this technique decreases the frequency of unanticipated admissions and readmissions after same-day surgery, together with their associated costs [33]. It has also been shown that the administration of nonsteroidal anti-inflammatory agents, such as tenoxicam [35] and diclofenac [29], and of opioids like morphine [36] leads to delay in the fracture healing process. However, the application of femoral block, leads to a decrease in the use of nonsteroidal anti-inflammatory agent and opioids for pain management at the perioperative period, and is beneficial from the fracture healing perspective.

In light of this study, we can postulate that, in the case of femoral fracture, the application of femoral nerve block, either once only or once a day for three consecutive days, had a positive impact via growth factors and WNT/ $\beta$-catenin signaling pathway activation, and histomorphometrically results in early fracture healing. Moreover, these effects accelerate the healing process of femoral fracture in rats.

\section{Acknowledgements}

This study was awarded with Best Free Paper by Turkish Regional Anesthesia Association at $13^{\text {th }}$ National Regional Association Congress, 2013.

\section{References}

1. Dimitriou R, Tsiridis E, Giannoudis PV. Current concepts of molecular aspects of bone healing. Injury. 2005;36:1392-1404. doi: 10.1016/j.injury.2005.07.019.

2. Einhorn TA. The science of fracture healing. J Orthop Trauma. 2005;19:4-6. PMID: 16479221.

3. Iwaki A, Jingushi S, Oda Y et al. Localization and quantification of proliferating cells during rat fracture repair: Detection of proliferating cell nuclear antigen by immunohistochemistry. J Bone Miner Res. 1997;12:96-102. PMID: 9240731.

4. Mackiewicz Z, Niklińska WE, Kowalewska J et al. Bone as a source of organism vitality and regeneration. Folia Histochem Cytobiol. 2011;49:558-569. doi. 10.5603/FHC.2011.0079.

5. Street J, Bao M, Guzman de L et al. Vascular endothelial growth factor stimulates bone repair by promoting angiogenesis and bone turnover. Proc Natl Acad Sci USA. 2002;23:9656-9661. doi: 10.1073/pnas.152324099.

6. Behonick DJ, Xing Z, Lieu S et al. Role of matrix metalloproteinase 13 in both endochondral and intramembranous ossification during skeletal regeneration. PLoS One. 2007;11:e1150. doi: 10.1371/journal.pone.0001150.

7. Azuma Y, Ito M, Harada Y et al. Low-intensity pulsed ultrasound accelerates rat femoral fracture healing by acting on the various cellular reaction in the fracture callus. J Bone $\mathrm{Mi}$ ner Res. 2001;16:671-680. doi: 10.1359/jbmr.2001.16.4.671. 
8. Guise TA. Molecular mechanisms of osteolytic bone metastases. Cancer. 2002;88:2892-2898. doi: 10.1002/10970142(20000615).

9. Jia J, Yao W, Amugongo S et al. Prolonged alendronate treatment prevents the decline in serum TGF- $\beta 1$ levels and reduces cortical bone strength in long-term estrogen deficiency rat model.Bone. 2013;52:424-432. doi:10.1016/j.bone.2012.10.017.

10. Loveridge N. Bone: more than a stick. J Anim Sci. 1999;77:190-196. doi: 10.2527/1999.77suppl_2190x.

11. Duvall CL, Taylor WR, Weiss D et al. Impaired angiogenesis, early callus formation, and late stage remodeling in fracture healing of osteopontin-deficient mice.J Bone Miner Res. 2007;22:286-297. doi: 10.1359/jbmr.061103.

12. Eriksen EF, Eghbali-Fatourechi GZ, Khosla S. Remodeling and vascular spaces in bone.J Bone Miner Res. 2007;22:1-6. doi: 10.1080/14041040701482935.

13. Wohl GR, Towler DA, Silva MJ. Stress fracture healing: Fatigue loading of the rat Ulna induces upregulation in expression of osteogenic and angiogenic genes that mimic the intramembranous portion of fracture repair. Bone. 2009;44:320-330. doi: 10.1016/j.bone.2008.09.010.

14. Baron R, Rawadi G. Minireview: Targeting the Wnt $/ \beta$ -catenin pathway to regulate bone formation in the adult skeleton. Endocrinology. 2007;148:2635-2643. doi: 10.1210/ en.2007-0270.

15. Chen Y, Whetstone HC, Lin AC et al. Beta-catenin signaling plays a disparate role in different phases of fracture repair: Implications for therapy to improve bone healing. PLOS Medicine 2007;4:1216-1229. doi: 10.1371/journal.pmed.0040249.

16. Ilfeld BM, Mariano ER, Madison SJ et al. Continuous femoral versus posterior lumbar plexus nerve blocks for analgesia after hip arthroplasty: a randomized, controlled study. Anesth Analg. 2011;113:897-903. doi: 10.1213/ ANE.0b013e318212495b.

17. Kardash K, Hickey D, Tessler MJ et al. Obturator versus femoral nerve block for analgesia after total knee arthroplasty. Anesth Analg. 2007;105:853-858. doi: 10.1213/01. ane.0000278158.36843.f7.

18. Koshy RC, Padmakumar G, Rajasree O. Low cost continuous femoral nerve block for relief of acute severe cancer related pain due to pathological fracture femur. Indian $J$ Palliat Care. 2010;16:180-182. doi: 10.4103/0973-1075.73669.

19. Le AX, Miclau T, Hu D et al. Molecular aspects of healing in stabilized and non-stabilized fractures. J Orthop Res. 2001;19:78-84. doi: 10.1016/S0736-0266(00)00006-1.

20. Raymond SA, Gissen AJ. Mechanism of differential nerve block. Hdbk Exp Pharmacol. Heidelberg: Springer-Verlag; 1987:95-164.

21. Colnot C, Thompson Z, Miclau T et al. Altered fracture repair in the absence of MMP9. Development. 2003;130:41234133. doi: $10.1242 /$ dev.00559.

22. Dempster DW, Compston JE, Drezner MK et al. Standardized nomenclature, symbols, and units for bone histomorphometry: a 2012 update of the report of the ASBMR histomorphometry nomenclature committee.J Bone Miner Res. 2013;28:1-16. doi: 10.1002/jbmr.1805.

23. Uslu S, Uysal A, Oktem G et al. Constructive effect of exogenous melatonin against osteoporosis after ovariectomy in rats. Anal Quant Cytol Histol. 2007;29:317-325. PMID: 17987812.

24. Filho ELR, Larrazabal MCA, Junior LFC et al. Effects of autologous stem cells on regenerated bone during distraction osteogenesis by Ilizarov technique in the radius of dogs. Histomorphometric analysis. Acta Cir Bras. 2013;28:574-581. doi. 10.1590/S0102-86502013000800004.

25. Edwards DS, Clasper JC. Heterotopic ossification: a systematic review. J R Army Med Corps. 2014;161:215-321. doi: 10.1136/jramc-2014-000277.

26. Vortkamp A, Pathia S, Perettib GM et al. Recapitulation of signals regulating embryonic bone formation during postnatal growth and in fracture repair. Mech Develop. 1998;71:65-76. PMID: 9507067.

27. Diniz SF, Amorim FPLG, Cavalcante-Neto FF et al. Alloxan-induced diabetes delays repair in a rat model of closed tibial fracture. Braz J Med Biol Res. 2008;41:373-379. doi: 10.1590/S0100-879X2008005000014.

28. Tatsuyama K, Maezawa Y, Baba $\mathrm{H}$ et al. Expression of various growth factors for cell proliferation and cytodifferentiation during fracture repair of bone. Eur J Histochem. 2000;44:269-278. PMID: 11095098.

29. Kanczler JM, Oreffo ROC. Osteogenesis and angiogenesis: the potential for engineering bone. Eur Cell Mater. 2008;15:100-114. PMID: 18454418.

30. Agholme F, Aspenberg P. Wnt signaling and orthopedics, an overview. Acta Orthop. 2011;82:125-130. doi: 10.3109/17453674.2011.572252.

31. Macfarlane AJR, Prasad GA, Chan VWS et al. Does regional anaesthesia improve outcome after total hip arthroplasty? A systematic review. Br J Anaesth. 2009;103:335-345. doi: 10.1093/bja/aep208.

32. Pagnano MW, Heb J, Horlocker T. Assuring a painless total hip arthroplasty: a multimodal approach emphasizing peripheral nerve blocks. J Arthroplasty. 2006;21:80-84. doi: 10.1016/j.arth.2006.03.001.

33. Klein SM, Evans H, Nielsen $\mathrm{KC}$ et al. Peripheral nerve block techniques for ambulatory surgery. Anesth Analg. 2005;101: 1663-1676. doi: 10.1213/01ANE.0000184187.02887.24.

34. Shum CF, Lo NN, Yeo SJ et al. Continuous femoral nerve block in total knee arthroplasty: immediate and two-year outcomes. J Arthroplasty. 2009;24:204-209. doi: 10.1016/j. arth.2007.09.014.

35. Grant SA, Nielsen KC, Greengrass RA et al. Continuous peripheral nerve block for ambulatory surgery. Reg Anesth Pain Med. 2001;26:209-214. doi: 10.1053/rapm.2001.22256.

36. Krischak GD, Augat P, Sorg T et al. Effects of diclofenac on periosteal callus maturation in osteotomy healing in an animal model. Arch Orthop Trauma Surg. 2007;127:3-9. doi: 10.1007/s00402-006-0202-x.

Submitted: 18 September, 2014

Accepted after reviews: 5 August, 2016

Available as AoP: 17 August, 2016 